\title{
ESTUDIO ELECTROFISIOLOGICO EN LA NEUROPATIA POR VINCRISTINA
}

\author{
Olga P. SANZ* \\ A. Colombi \\ M. Somoza \\ D. MONTEVERDE
}

El efecto tóxico de la vincristina y vinblastina sobre el nervio periférico es una de las complicaciones que más asiduamente se observa desde la introducción terapéutica de estas drogas como agentes citotóxicos ${ }^{1,13}$.

Varios autores han analizado el tema desde ángulos diferentes observando una serie de hechos que apuntan hacia la presencia de participación del sistema nervioso en pacientes tratados con estas drogas ${ }^{4,5}, 17,19$. Se describen como alteraciones precoces: pérdida de los reflejos tendinosos, generalmente acompañada de parestesias y disminución de la fuerza muscular. Bradley y col. ${ }^{4,5}$ observaron cambios electromicroscópicos en músculos proximales con disminución de su fuerza en pacientes en los cuales no habia evidencias electrofisiológicas de desorden muscular.

Por lo tanto, de acuerdo a los autores citados, estas drogas tendrían más de un efecto a nivel fisiológico siendo dificil arrojar conclusiones sobre sus mecanismos de acción; sin embargo existen pruebas suficientes como para aceptar la acción de estos fármacos sobre el flujo axoplásmico. Bensch y Malawista ${ }^{2,3}$ observaron que la vinblastina y la vincristina interactúan con las proteinas microtubulares formando agregados paracristalinos interrumpiendo de esta manera el flujo trófico.

El presente trabajo analiza, por métodos electrofisiológicos, el compromiso de la unidad motora en pacientes intoxicados con vincristina.

\section{MATERIAL Y METODOS}

Se estudiaron dos mujeres y ocho hombres cuyas edades oscilaron entre $10 \mathrm{~s} 28$ y 57 años, con sintomas y signos de neuropatia periférica. Todos ellos se hallaban en tratamiento con vincristina en el momento del examen. El tiempo de recepción de la droga varió entre 1 y 36 meses; la dosis recibida osciló entre 7 y $48 \mathrm{mg}$.

Sección de Electroneurofisiología Clínica del Policlínico "Dr. José M. Ramos Mefia": *Becaria de Investigación de la Municipalidad de la Ciudad de Buenos Aires.

Agradecimiento - Los autores agradecen al Dr. R. E. P. Sica sus sugerencias y valiosas criticas a este trabajo. 
Se piocedió en la primera fase del estudio a cuantificar el número de unidades motoras (UM) funcionantes en los músculos de la eiminencia tenar y a medir el incremento individual de cada una de las primeras 8-10 UM reclutadas por el estímulo graduado de intensidad creciente sobre el nervio mediano, siguiendo el método descripto por Sica, McComas, Upton y Longmire (1974) ${ }^{20}$.

La segunda fase del exámen tuvo como objeto la medición de la velocidad de conducción motora y latencia residual en el nervio mediano; para ello se siguió la lécnica descripta por Hodes, Larrabee y German (1948) ${ }^{12}$, utilizando un impulso cuadrado de $0,5 \mathrm{~ms}$ de duración con intensidad supramáxima para la respuesta muscular evocada, liberado desde un electroestimulador Multistin modelo 13GO4.

Junto al exámen mencionado se estudiaron los valores de velocidad de conducción sensitiva del mismo nervio por impulso ortodrómico de las fibras digitales del dedo medio, registrando el potencial sensitivo compuesto del mencionado nervio a nivel de la muñeca, con electrodos de superficie. Siete pacientes fueron sometidos a esta investigación.

En la última etapa del estudio se procedió a la estimulación repetitiva del nervio mediano con estímulos de $0.5 \mathrm{~ms}$ de duración a intensidad supramáxima para la respuesta motora a frecuencias de 3,10 y $30 \mathrm{~Hz}$. El registro de la actividad evocada se hizo en los músculos de la eminencia tenar.

Todos los registros fueron efectuados con electrodos de superficie. Los potenciales obtenidos fueron amplificados a través de un amplificador Hewllet-Packard modelo 1403A y medidos en un osciloscopio Hewllet-Packard con depósito modelo $141 \mathrm{~A}$.

Lus valores obtenidos en el grupo probante fueron controlados con grupos testigos. El número de controles para la cuantificación de UM fué de 35 sujetos por debajo de los 60 años de edad. Para la velocidad de conducción y latencia residual motora del nervio mediano 48 sujetos y, para la sensitiva del mismo nervio, 40 sujetos.

Tratamiento de los resultados - La significación entre las medias fue valorada por el test " $\mathrm{t}$ " de Student. A través del texto los valores medios se dan con su desviación standard simple.

\section{RESULTADOS}

El número de UM funcionantes estimado en los músculos de la eminencia tenar, en el lote de pacientes, fue significativamente inferior al encontrado en el grupo testigo (control $318 \pm 71 \mathrm{UM}$; probante $174 \pm \mathrm{UM} ; \mathrm{P}<0.001$ ). El tiempo de recepción de la droga en los pacientes estudiados con este método no guardó correlación con la pérdida de UM observada (coeficiente de correlación: 0,07). Efectuando la correlación entre el número de UM remanentes y la dosis recibida hasta el momento del exámen no se obtuvo tampoco significación (coeficiente de correlación: 0.01 ).

El tamaño medio de UM comparado con el lote control fue significativamente menor (control $40.42 \pm 14.53 \mathrm{uv}$; probante $33.60 \pm 19.20 \mathrm{uv} ; \mathbf{P}<0.001$ ). Sin embargo, tomando los valores de los tamaños individuales correspondientes a cada UM, en ambos lotes, se observó que un alto porcentaje de UM en los individuos afectados se ubicó a la izquierda del histograma mostrando elevada incidencia de UM de tamaño reducido; junto a ellas se observaron otras unidades cuyo tamaño correspondia a la del lote control, dibujando un histograma bimodal, hecho que demuestra la coexistencia de UM de tamaño disminuido junto a otras de caracteres normales (Grafico 1).

El exámen de la velocidad de conducción y su latencia residual de las fibras motoras rápidas del nervio mixto, mostró, comparando las medias aritméticas de uno y otro parámetro con sus similares controles, la existencia de diferencias significativas con cifras de velocidades de conducción disminuídas (control 58.27 \pm 5.24 $\mathrm{m} / \mathrm{sg}$; probante $46.95 \pm 6.88 \mathrm{~m} / \mathrm{seg}$; $\mathrm{P}<0.0005$ ) y latencias residuales prolongadas (control $2.95 \pm 0,56 \mathrm{~ms}$; probante $4.15 \pm 0,65 \mathrm{~ms} ; \mathrm{P}<0.0005$ ). 


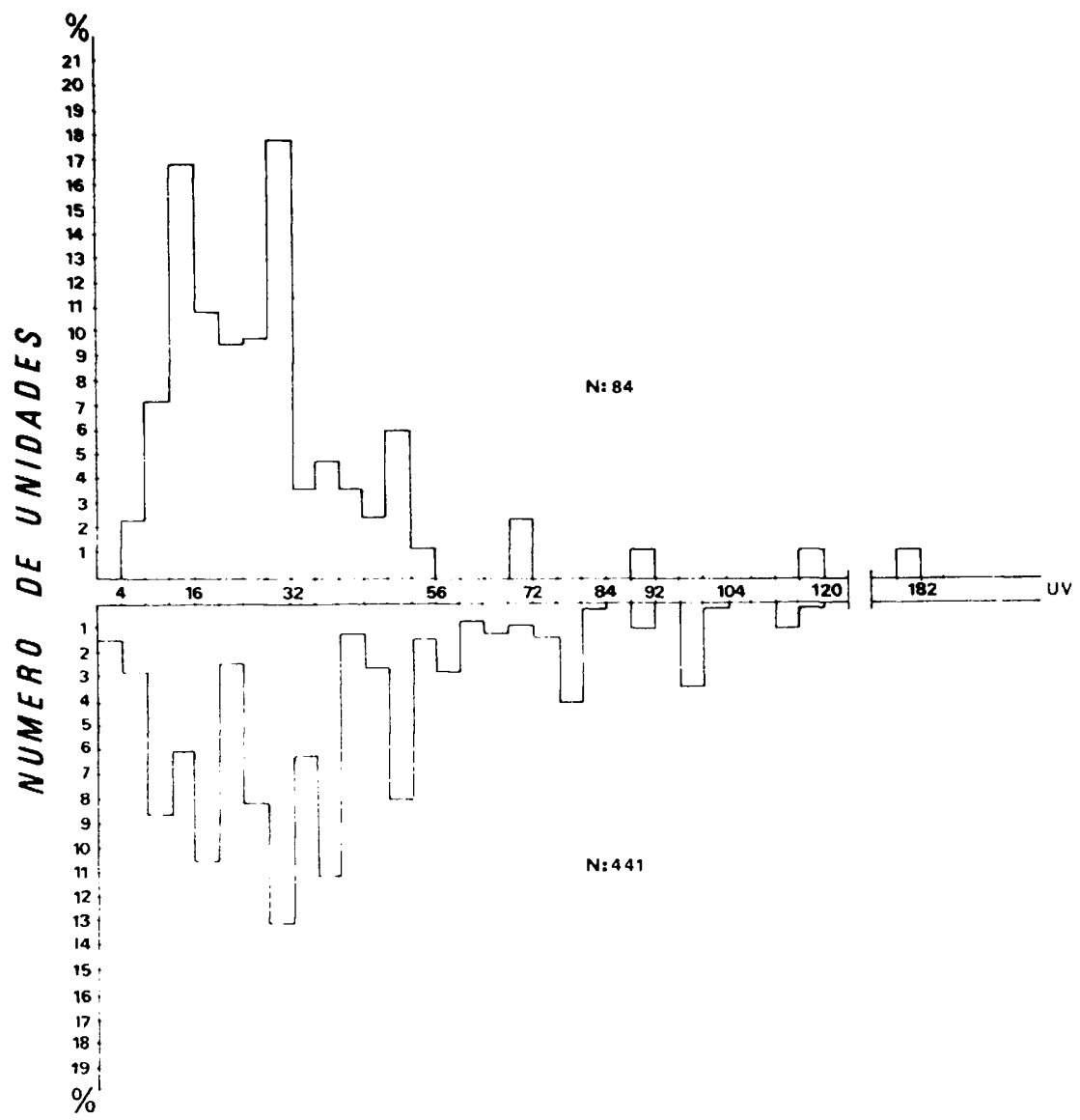

Grafico 1 - Valores individuales de la magnitud de unidade motora. En la parte superior, probante; en la parte inferior, control $(N=n u$ mero de potenciales de $U M ; \mu v=$ microvoltios).

Los valores medios de la velocidad de conducción sensitiva del nervio mediano y la amplitud del potencial sensitivo del mismo nervio arrojó cifras significativamente menores que las correspondientes al grupo control (control $55.12 \pm 4.70 \mathrm{~m} / \mathrm{seg}$.; probante $37.36 \pm 11.10 \mathrm{~m} / \mathrm{seg}$; $\mathrm{P}<0.005$ ). Amplitud, control: $31.75 \pm 13.05 \mathrm{uv}$; probante: $8.86 \pm 5.50$ uv; $P<0.0005)$. Se puede observar que las cifras de velocidad de conducción sensitiva en los pacientes se ubicaron en su mayoria cercanas al limite inferior de la normalidad; en tanto que los valores de la amplitud del potencial sensitivo se hallaban en alta proporción por debajo del limite inferior de la normalidad (Gráfico 2 y Tabla 1 ).

En el $57 \%$ de los casos estudiados se observó la caída de la magnitud del potencial de acción muscular evocado por estímulo repetitivo del nervio mediano, hecho que indica la participación de la placa motora en el trastorno. 

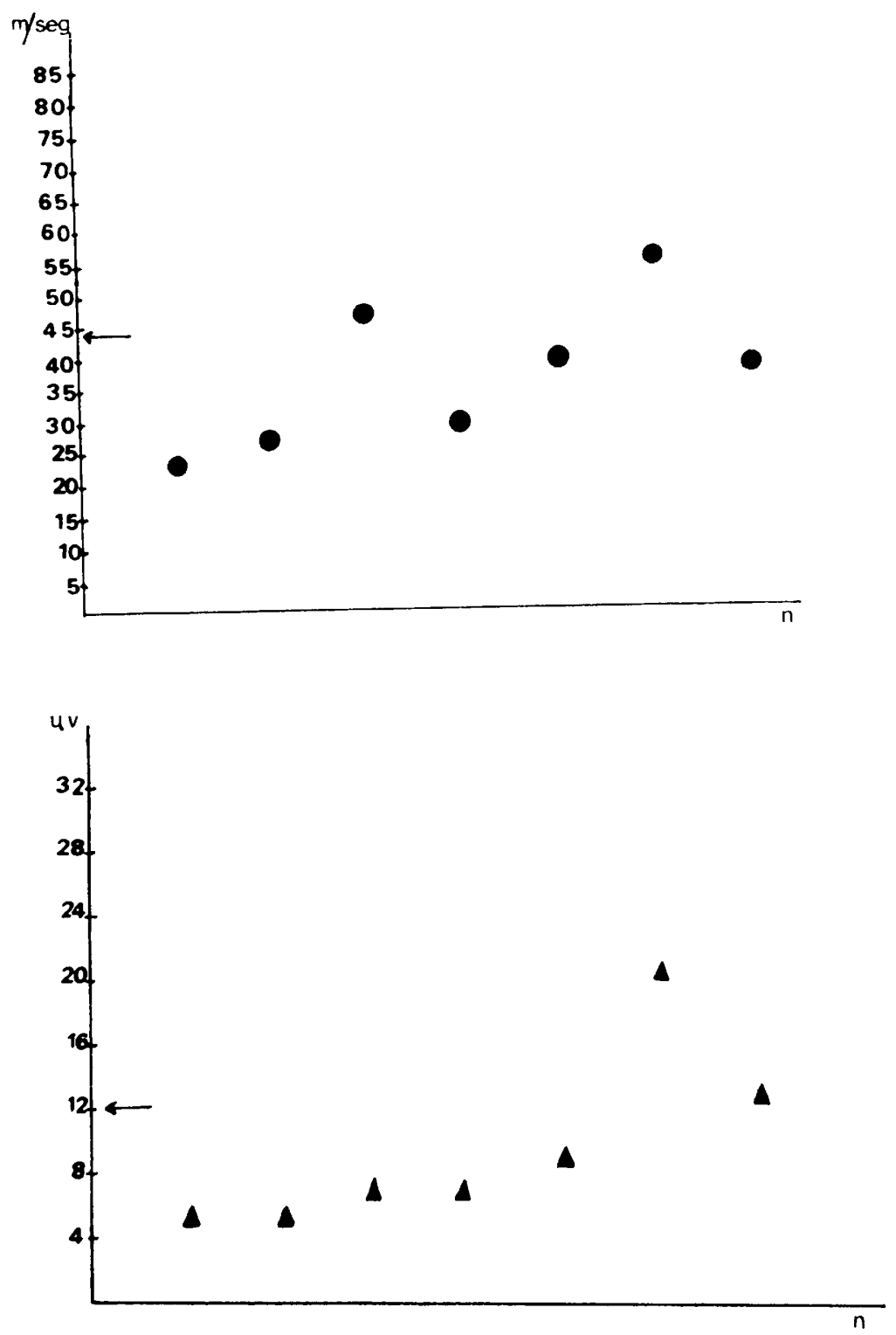

Grafico 2 - En la parte superior, velocidad de conducción sensitiva del nervio mediano ( $n=$ numero de pacientes; $\mathrm{m} / \mathrm{seg}$. $=$ - metros por segundo). En la parte inferior, amplitud del potencial sensitivo del nervio mediano ( $n=$ numero de pacientes; $\mu v=$ microvoltios). La flecha sobre las ordenadas indica el limite inferior de los valores correspondientes al grupo control. 


\begin{tabular}{crcccc}
\hline Paciente & $\mathrm{n}$ & $\begin{array}{c}\text { V. de C.m } \\
(\mathrm{m} / \mathrm{seg})\end{array}$ & $\begin{array}{c}\text { Lat. d.m } \\
(\mathrm{ms})\end{array}$ & $\begin{array}{l}\text { V. de C.s } \\
(\mathrm{m} / \mathrm{seg})\end{array}$ & $\begin{array}{c}\text { Amp. Pot.s } \\
(\mu \mathrm{v})\end{array}$ \\
\hline C.A. & 113 & 40 & 4.5 & 24.5 & 5 \\
B.S. & 56 & 48 & 4.2 & 27 & 5 \\
F.L. & 300 & 35 & 3.4 & & 6 \\
A.B. & 217 & 44 & 4.2 & 47 & 6 \\
M.F. & 178 & 52 & 4.6 & & 8 \\
R.L. & 193 & 42,5 & 5 & 30 & 20 \\
A.B. & 175 & 52 & 4.4 & 40 & 12 \\
S.M. & 85 & 51 & 4.1 & 55 & 12 \\
B.M. & 122 & 59 & 2.7 & & 5 \\
G.M. & 306 & 46 & 4.4 & 38 & 74 \\
Control & 220 & $>50$ & $<4.6$ & $>44$ & $>12$ \\
\hline
\end{tabular}

Tabla 1 - Valores individuales en pacientes tratados con vincristina. Leyenda: $n=$ numero de UM; V. de $C . m=$ velocidad de conducción motora; Lat. d.m = latencia distal motora; $V$. de C.s = velocidad de conducción sensitiva; Amp. Pot.s = amplitud potencial sensitivo; $m / s e g .=m e t r o$ por segundo; $m s=$ milisegundos; $\mu v=$ microvoltios.

\section{DISCUSION}

Los resultados aquí presentados corroboran el compromiso del aporte nervioso al músculo en los pacientes bajo tratamiento con vincristina. Un hecho en el que esta conclusión se ha basado es la pérdida significativa de UM. Teniendo en cuenta la hipótesis que se ha dado en llamar "de la motoneurona enferma" 15 las estructuras de ella dependientes en cuanto a su efecto trófico se refiere, tanto el axón como las fibras musculares que ese axón inerva, sufren las consecuencias de aquel deterioro pudiendo llegar a su degeneración. Las manifestaciones de la dificultad en mantener una conexión sináptica adecuada con las fibras musculares que inerva son: reducción de la amplitud del potencial evocado por estimulación repetitiva (respuesta miasteniforme), bajos valores de velocidad de conducción motora marcados sobre todo en los últimos tramos del axón ${ }^{7}$, siendo un hecho más demostrativo la imposibilidad de hacer crecer colaterales axónicas y tomar bajo su tutela una proporción de fibras musculares huérfanas de inervación.

Analizando este último hecho en forma más detenida es posible suponer que la motoneurona enferma pierde fibras musculares correspondientes a su unidad motora, situación que lleva a la disminución del tamaño de esa unidad. Este concepto tiene su paralelo con la concepción de Engel (1973) ${ }^{8}$ de la llamada denervación "in portio" y explica en nuestros hallazgos la presencia de unidades motoras de tamaño reducido que dibujan el primer modo del histograma mostrado en el gráfico 1. Aceptando este criterio es 
razonable pensar que muchas de las fibras musculares integrantes de estas unidades motoras quedarán desprovistas de inervación y podrian ser adoptadas por motoneuronas sanas, mecanismo a través del cual aumentarían su tamaño. Sin embargo observando el histograma del grafico 1 no se ve en el grupo probante UM cuyo tamaño supere el del modo control, hecho entonces que sugiere la incapacidad de las Um que constituyen el segundo modo para adquirir nuevas fibras por ramificación colateral, situación que inclina a pensar que existe ya en esas neuronas un estado de enfermedad, una de cuyas primeras manifestaciones es la arriba mencionada.

Observando los valores de velocidad de conducción motora y sensitiva obtenidos en el grupo probante se vuelve aparente el mayor compromiso en los segmentos distales. Este hallazgo junto a las cifras de velocidad de conducción obtenidas que no son lo suficientemente bajas como para aceptar la presencia de desmielinización segmentaria ${ }^{9}$ lleva, sumado a lo dicho en relación a los cambios de UM y a la respuesta miasteniforme obtenida en un porcentaje de estos pacientes, a la conclusión de que la droga aqui empleada produce cambios primariamente a nivel axónico. Esta alteración ha sido ya descripta por otros autores $2,3,4,5,6,10,11,13,14.17,18.19,21$ aunque ninguno de ellos, hasta donde nuestra información alcanza, describió los cambios dinámicos observados en el comportamiento de la unidad motora. En toda la bibliografía revisada hemos podido encontrar una sola observación que en cierta forma puede asimilarse a la nuestra, que es la hecha por McComas. Sica, Upton y Petito (1973) ${ }^{16}$ en un solo caso de intoxicación por vincristina.

En nuestro material no ha existido correlación entre la dosis empleada hasta el momento del exámen $\mathrm{y} / \mathrm{o}$ el tiempo de tratamiento, con el daño del sistema nervioso periférico visto; sin embargo esta observación tiene la limitación de ser estática en cuanto a que no ha sido seguido el curso de la intoxicación en estos pacientes por lo que sería aventurado extraer conclusiones de tal hallazgo.

Un último hecho merece el comentario, cual es la disminución de la amplitud del potencial sensitivo evocado. La caída de tal potencial, en relación a los controles, debe ser atribuída a la pérdida de fibras sensitivas que contribuyen a la formación de dicho potencial; pero si por otro lado se piensa que la velocidad de conducción sensitiva no alcanza valores que justificaran la aceptación de desmielinización segmentaria ", esa disminución debe ser atribuída a la pérdida funcional de fibras de bajo diámetro y por lo tanto de baja velocidad de conducción.

\section{RESUMEN}

Diez pacientes afectados por diversas patologías que requerian tratamiento crónico con Vincristina, fueron sometidos a estudios electrofisiológicos en los que se valoró: el número de unidades motoras (UM) funcionantes en los músculos de la eminencia tenar, los valores de los incrementos 
medios de UM, velocidad de conducción motora y su latencia residual en el nervio mediano, la velocidad de conducción sensitiva del mismo nervio y el estado de la transmisión neuromuscular. Los valores obtenidos fueron comparados con grupos controles.

Los resultados mostraron disminución del número de UM; las UM remanentes presentaron amplitud reducida junto a otras cuyo tamaño no superaba el del grupo control, hecho que sugiere la incapacidad de lograr una reinervación adecuada. Las velocidades de conducción motora y sensitiva mostraron valores diminuídos, con mayor compromiso en los segmentos distales. Junto a estos datos se halló respuesta miasteniforme al estímulo repetitivo. Todos estos resultados permiten postular la existencia de un compromiso de la unidad motora, abarcando todos sus segmentos, en pacientes intoxicados con Vincristina.

\section{SUMMARY}

Vincristine neuropathy: an electrophysiological study

Ten patients treated with vincristine were submitted for electrophysiological examination. It was investigated the number of motor units within the thenar muscle following a technique described previously (Sica et al. 1974); motor and sensitive conduction velocities as well as motor distal latencies in the median nerve were studied following conventional techniques. The behaviour of the evoked muscle potential with repetitive supramaximal stimulation over the median nerve was also investigated. The findings were compared with control groups.

The estimated number of motor units was disminished in eight of ten patients and the average number was significantly different from the control group (control $318 \pm 71 \mathrm{UM}$; patients $174 \pm 84 \mathrm{UM} ; \mathrm{P}<0.001$ ). The potential amplitudes in most of the surviving units were reduced, others remaining within the normal range. This makes apparent that the peripherical nervous system fails to compensate adequatly and, furthermore, a loss of individual muscle fibres occurs within some individual units. The conduction velocities of the fastest conducting motor nerve fibres were reduced and motor distal latencies prolongued (Table 1). Maximal impulse conduction velocities were measured in sensory fibres. In 5 of 7 subjets investigated the values laied just beyond the lower limit of the normal range. The amplitude of the sensory orthodromic evoked potential in the median nerve at the wrist was disminished almost in the whole group. The decremental muscle response to repetitive nerve stimulation, can be interpreted as the result of the damage at the neural apparatus at the motor end plate; it was observed in $57 \%$ of the patients. In summary, evidences have been registered showing that the nervous supply to the muscle is affected in patients treated with vincristine; the motor unit behaviour under this conditions is discussed. 


\section{REFERENCIAS}

1. ARMSTRONG, J. G.; DYKE, R. W. \& FOUTS, P. J. - Initial clinical experience with leurocristine, a new alkaloid from Vinca rosea Linn. Proc. Am. Ass. Cancer Res. 3:301, 1962 .

2. BENSCH, K. G. \& MALAWISTA, S. E. - Microtubule crystals: a new biophysical phenomenon induced by Vinca alkaloids. Nature 218:1176, 1968.

3. BENSCH, K. G. \& MALAWISTA, S. E. - Microtubular crystals in mammalian cells. J. Cell Biol. 40:95, 1969.

4. BRADLEY, W. G. - The neuromyopathy of vincristine in the guinea-pig: an electrophisiological and pathological study. J. Neurol. Sci. 10:133, 1970.

5. BRAdley, W. G.; LASSMAN, L. P.; PEARCE, G. W. \& WALTON, J. N. - The neuromyopathy of vincristine in man: clinical, electrophysiological and pathological study. J. Neurol. Sci. 10:107, 1970.

6. CASEY, E. B.; JELLIFE, A. M.; LE QUESNE, P. M. \& MILLETT, Y. L. - Vincristine neuropathy: clinical and electrophysiological observations. Brain 96:69, 1973.

7. CAVANAGH, J. B. - The significance of the "dying back" process in experimental and human neurological disease. Int. Rev. exp. Path. 3:219, 1964.

8. ENGEL, W. K. \& WARMOLTS, J. R. - The motor unit. New Developments in Eletromyography and Clinical Neurophysiology 1:141, 1973.

9. GILliAT, R. W. - Nerve conduction in human and experimental neuropathies. Proc. R. Soc. Med. 59:989, 1966.

10. GOTTSCHALK, P. G.; DYCK, P. J. \& KIELY, J. M. - Vinca alkaloid neuropathy: nerve biopsy studies in rats and in man. Neurology (Minneapolis) 18:875, 1968.

11. HILDEBRAND, J. \& COERS, C. - Etude clinique, histologique et électrophysiologique des neuropathies associées au traitement par la vincristine. Eur. J. Cancer 1:51, 1965.

12. HODES, R.; LARRABEE, M. C. \& GERMAN, W. - The human electromyogram in response to nerve stimulation and the conduction velocity in motor axons. Arch. Neurol. Psychiat. (Chicago) 60:340, 1948.

13. KARON, M. - Leurocristine sulfate in the treatment of acute leukemia. Proc. Am. Ass. Cancer Res. 3:333, 1962.

14. MARANTZ, R.; VENTILLA, M. \& SHELANSKI, M. - Vinblastine-induced precipitation of microtubule protein. Science 165:498, 1969.

15. McCOMAS, A. J.; SICA, R. E. P. \& CAMPBELL, M. J. - Sick motoneurones: a unifying concept of muscle disease. Lancet I:321, 1971.

16. McCOMAS, A. J.; SICA, R. E. P.; UPTON, A. R. M. \& PETITO, F. - Sick motoneurones and muscle disease. Ann. New York Acad. Sci. 228:261, 1973.

17. MCLEOD, J. G. \& PENNY, R. - Vincristine neuropathy: an electrophysiological and histological study. J. Neurol. Neurosurg. Psychiat. 32:297, 1969.

18. SAMSON Jr., F. E. - Mechanism of axoplasmic transport. J. Neurobiology $2: 347,1971$.

19. SANDLER, S. G.; TOBIN, W. \& HENDERSON, E. S. - Vincristine-induced neuropathy: a clinical study of fifty patients. Neurology (Minneapolis) 19:367, 1969.

20. SICA, R. E. P.; McCOMAS, A. J.; UPTON, R. M. \& LONGMIRE, D. - Number of motor units in small muscles of the hand. J. Neurol. Neurosurg. Psychiat. $37: 55,1974$.

21. WILSON, L.; BRYAN RUBY, A. \& MAZIA, L. - Precipitation of proteins by vinblastine and calcium ions. Proc. Nat. acad. Sci. 66:807, 1970.

Sección de Electroneurofisiología Clínica - Hospital J. M. Ramos Mejía - Urquiza 609 - Buenos Aires - Argentina. 